\title{
Effect of a polyphenol-rich dietary pattern on intestinal permeability and gut and blood microbiomics in older subjects: study protocol of the MaPLE randomised controlled trial
}

Simone Guglielmetti ${ }^{1 \dagger}$, Stefano Bernardi ${ }^{1 \dagger}$, Cristian Del Bo ${ }^{\prime 1}$, Antonio Cherubini ${ }^{2}$, Marisa Porrini ${ }^{1}$, Giorgio Gargari ${ }^{1}$, Nicole Hidalgo-Liberona ${ }^{3,4}$, Raul Gonzalez-Dominguez ${ }^{3,4}$, Gregorio Peron ${ }^{3,4}$, Raul Zamora-Ros ${ }^{3,5}$,

Mark S. Winterbone ${ }^{6}$, Benjamin Kirkup ${ }^{6}$, Paul A. Kroon ${ }^{6}$, Cristina Andres-Lacueva ${ }^{3,4}$ and Patrizia Riso ${ }^{1 *}$ (D)

\begin{abstract}
Background: During aging, alterations of the intestinal microbial ecosystem can occur contributing to immunosenescence, inflamm-aging and impairment of intestinal barrier function (increased intestinal permeability; IP). In the context of a diet-microbiota-IP axis in older subjects, food bioactives such as polyphenols may play a beneficial modulatory role.

Methods: MaPLE is a project centered on a randomized, controlled cross-over dietary intervention trial [polyphenol-rich diet (PR-diet) versus control diet (C-diet)] targeted to older people ( $\geq 60 \mathrm{y}$ ) living in a wellcontrolled setting (i.e. nursing home). The 8-week interventions are separated by an 8-week wash-out period. Three small portions per day of selected polyphenol-rich foods are consumed during intervention in substitution of other comparable products within the C-diet. Biological samples are collected before and after each treatment period to evaluate markers related to IP, inflammation, vascular function, oxidative stress, gut and blood microbiomics, metabolomics. A sample size of 50 subjects was defined based on IP as primary outcome.

Discussion: Evidence that increasing the consumption of polyphenol-rich food products can positively affect intestinal microbial ecosystem resulting in reduced IP and decreased translocation of inflammogenic bacterial factors into the bloodstream will be provided. The integration of data from gut and blood microbiomics, metabolomics and other IP-related markers will improve the understanding of the beneficial effect of the intervention in the context of polyphenols-microbiota-IP interactions. Finally, findings obtained will provide a proof of concept of the reliability of the dietary intervention, also contributing to future implementations of dietary guidelines directed to IP management in the older and other at risk subjects.
\end{abstract}

Trial registration: The trial is registered at (ISRCTN10214981); April 28, 2017.

Keywords: Gut barrier function, Leaky gut, Flavonoids, Phenolics, Inflammation, Aging, Inflamm-aging

\footnotetext{
* Correspondence: patrizia.riso@unimi.it

†Simone Guglielmetti and Stefano Bernardi contributed equally to this work.

${ }^{1}$ Department of Food, Environmental and Nutritional Sciences (DeFENS),

Università degli Studi di Milano, 20133 Milan, Italy

Full list of author information is available at the end of the article
}

(c) The Author(s). 2020 Open Access This article is distributed under the terms of the Creative Commons Attribution 4.0 International License (http://creativecommons.org/licenses/by/4.0/), which permits unrestricted use, distribution, and reproduction in any medium, provided you give appropriate credit to the original author(s) and the source, provide a link to the Creative Commons license, and indicate if changes were made. The Creative Commons Public Domain Dedication waiver (http://creativecommons.org/publicdomain/zero/1.0/) applies to the data made available in this article, unless otherwise stated. 


\section{Background}

Age-associated changes significantly compromise health status and increase the risk of chronic diseases. Within these modifications, recent research has been focusing on those that specifically occur at the gut epithelium level with impact on intestinal immune homeostasis and related systemic responses [1]. The maintenance of a functional intestinal barrier (the functional entity separating the gut lumen from the inner host) [2], seems to be of utmost importance to facilitate healthy aging. Nevertheless, no conclusive evidence exists for a direct or causal link between the aging process and intestinal mucosa integrity impairment $[3,4]$.

The intestine acts both as a barrier (to keep harmful substances out of the body) and as a selectively permeable surface that allows the controlled passage of substances from the gut lumen through the gut wall and into the body. This controlled flux across the intestinal wall is known as intestinal permeability (IP) [2]. Inappropriate IP (i.e. loss of control of the influx of substances from the gut) has been associated with several disorders and diseases, such as irritable bowel syndrome, inflammatory bowel disease, allergy, colon cancer, obesity, celiac disease, inflammatory joint diseases and neurologic pathologies (e.g. Parkinson's disease) [5-8]. In this regard the intestinal microbiota is considered an important factor in the regulation of IP, in fact, gut microorganisms may directly affect IP through tight junction modulation [9] and indirectly by contributing to the up/ down regulation of inflammatory processes, which is a key factor in causing impaired IP [10]. Consequently, manipulation of the complex intestinal microbial ecosystem (i.e. the microbiota and derived metabolic products) has been proposed as a novel strategy to maintain/improve normal IP function [2].

Increasing evidence suggests that dietary patterns can represent a relevant factor in shaping the intestinal microbiota and modifying the relative abundance of specific bacterial taxa [11-13]. Consequently, modulating the concentrations of health-affecting microbial metabolites in the gut such as butyrate $[14,15]$, has been suggested to preserve tight junction integrity and inhibit TNF-alpha release, thus maintaining appropriate IP condition [16]. Nutrients are also essential themselves and malnutrition is associated with increased IP [17].

Older subjects are often characterized by alterations of the intestinal microbial ecosystem $[18,19]$, which may be due to inadequate nutrition, drug treatments and other age-related factors: all of these seem to contribute to immunosenescence and inflamm-aging $[18,20]$.

In the context of a diet-microbiota-IP axis, food bioactives may have a key role in regulating the numerous interconnected processes involved. Particularly, polyphenols exert antioxidant, anti-inflammatory/immunomodulatory properties at intestinal and systemic levels, and there is increasing mechanistic evidence suggesting their potential to modulate IP [21, 22]. In addition, polyphenols are extensively metabolized by the microbiota and can affect its composition [13, 23]. The combination of the modulation of intestinal ecology by polyphenols and the effect on derived microbial metabolites has been shown to improve inflammatory markers [24]. Taken together, these data support findings obtained from observational studies in older subjects suggesting that a high polyphenol diet is associated with favorable health outcomes [25]. But, wellcontrolled intervention studies are still lacking [21].

\section{Aim}

The aim of the MaPLE project (Microbiome mAnipulation through Polyphenols for managing Leakiness in the Elderly) is to evaluate the hypothesis that an increased intake of polyphenol-rich foods can reduce IP and lower inflammogenic bacterial factors in the bloodstream promoting an overall protective/beneficial metabolic phenotype in older subjects. Three approaches have been taken; the main study, a dietary intervention randomized controlled trial described here, combined with preclinical studies in an animal model of aging to test the impact of the polyphenol-rich diet on IP associated markers, and also in cultured human intestinal cells (caco-2) to investigate the capacity of single polyphenols to modulate IP.

\section{Methods/design \\ MaPLE RCT: protocol and study design}

The MaPLE RCT is a single-blind, randomised, controlled, cross-over trial [polyphenol-rich diet (PR-diet) versus control diet $(C$-diet)] in older people $(\geq 60$ y) living in a nursing home. Each intervention period consists of 8 weeks and is separated by an 8-week wash-out period in which participants consume their habitual diet to avoid carry-over effects.

The PR-diet and C-diet were developed to provide adequate and comparable levels of energy and nutrients. The PR-diet was achieved by replacing three portions per day of low polyphenol foods/beverages with specific polyphenol-rich foods/beverages (as detailed below). During the study, subjects are asked to fast overnight before each scheduled time-point of blood, urine and feces collection. In addition, daily menus and weighted food records (WFRs) are collected throughout the trial. An overview of the study design is represented in Fig. 1 and Table 1. The study adhered to SPIRIT guidelines.

\section{Trial status}

The trial has been prospectively registered (April 28, 2017; ISRCTN10214981). 


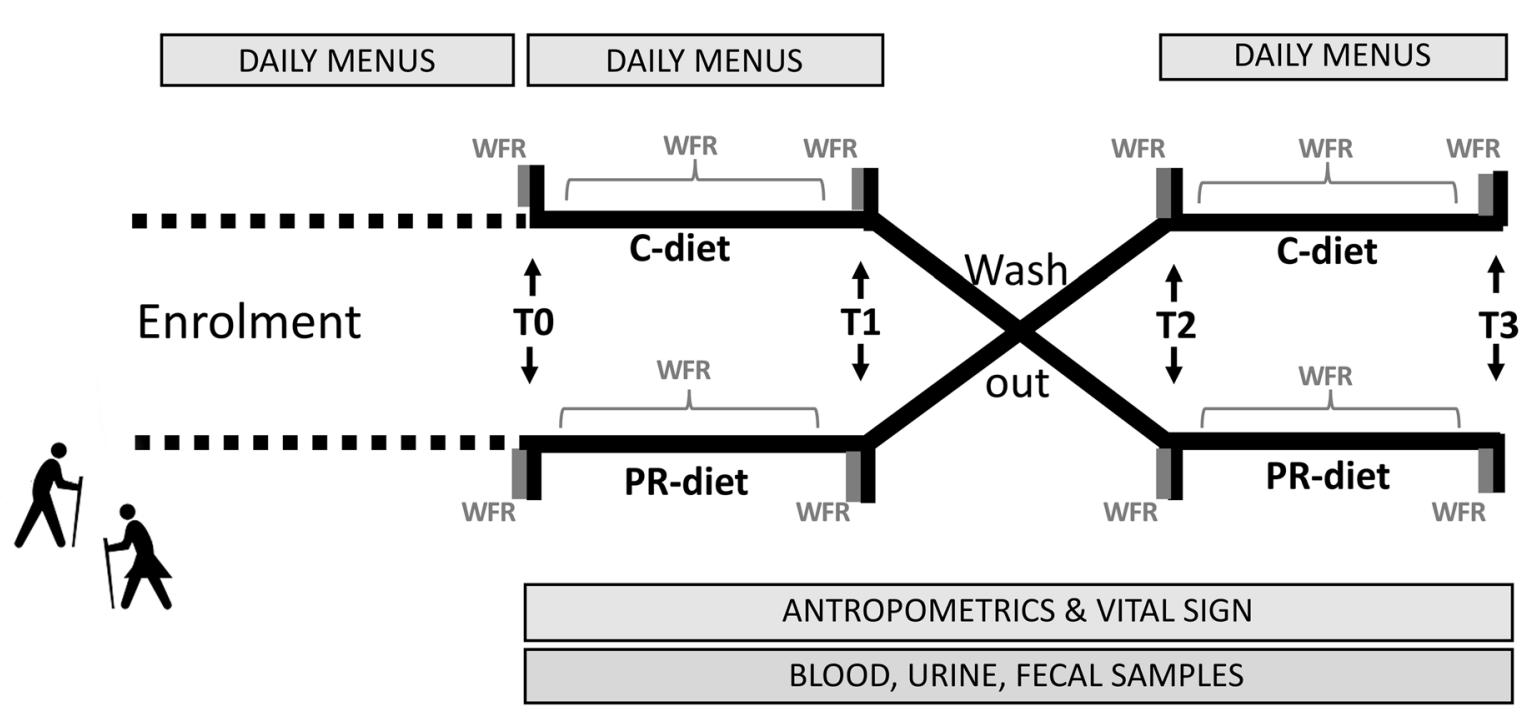

Fig. 1 Study design: Schematic representation of the study workflow. WFR = weighed food records; T0, T1, T2, T3 = time of intervention; C-diet = control diet PR-diet $=$ polyphenol-rich diet

The whole trial has been completed (December 2019); analyses and data elaboration are still ongoing.

\section{Location}

The intervention has been performed at Civitas Vitae (OIC Foundation, Padua, Italy) which hosts a large number of older people living in residential care buildings or in independent residences located in the same area, depending on individual willingness and level of disabilities. The OIC Foundation provides several facilities and dedicated area for meal preparation. This allows to collect accurate information with regard the composition of the diets from the recipes used for each of the foods in the meals delivered daily to the participants. We were able to accurately assess food intake using weighed food records in the intervention study.

\section{Participant enrollment}

Before recruitment, a meeting with the medical staff and nurses' coordinators at OIC Foundation took place in order to present and widely discuss the aim, methodologies and technical aspects related to the development and the management of the MaPLE RCT. After this meeting, several formal presentations of the project aim and some general information on the intervention planned were organized at OIC Foundation for the hosts and their families. Finally, an accurate evaluation of the host characteristics was performed in collaboration with the physicians/geriatricians and nurses' coordinators to pre-select based on the verification of the main inclusion and exclusion criteria (see below) and to identify plausible candidates for the study. Subjects who were interested in participating in the study signed an informed consent reporting all the information on the dietary intervention, the analysis and protocols that they were asked to undertake/follow.

More specifically, volunteers were selected according to the inclusion and exclusion criteria reported below:

\section{Inclusion criteria}

- Age $\geq 60$ years

- Adequate nutritional status evaluated with Mini Nutritional Assessment (MNA), score $\geq 24$

- Good cognitive status tested with Mini Mental State Examination (MMSE), score $\geq 24$

- Self-sufficiency assessed with validated tests (e.g. Barthel index - activities of daily living, score $\geq 60$ )

- Increased intestinal permeability evaluated by serum zonulin level

\section{Exclusion criteria}

- Celiac disease

- Severe liver disease with cirrhosis

- Severe renal insufficiency (dialysis)

- Presence of severe Chronic Obstructive Pulmonary Disease (COPD; oxygen therapy for many hours a day) or severe cardiovascular disease (heart failure 
Table 1 Standard protocol items: recommendations for interventional trials (SPIRIT)

\begin{tabular}{|c|c|c|c|c|c|}
\hline & ENROLLMENT & TO & T1 & T2 & T3 \\
\hline \multirow{3}{*}{$\begin{array}{l}\text { Eligibility screening } \\
\text { Informed consent } \\
\text { Intestinal Permeability test }\end{array}$} & $x$ & & & & \\
\hline & $x$ & & & & \\
\hline & $x$ & & & & \\
\hline Daily menu evaluation & $\mathrm{x}$ & & & & \\
\hline Polyphenol intake & $x$ & & & & \\
\hline \multicolumn{6}{|c|}{ PHYSICAL ASSESSMENT AND VITAL SIGN } \\
\hline Anthropometrics & & $\mathrm{x}$ & $x$ & $x$ & $\mathrm{x}$ \\
\hline Blood pressure & & $\mathrm{X}$ & $x$ & $\mathrm{X}$ & $x$ \\
\hline \multicolumn{6}{|c|}{ DIETARY ASSESSMENTS } \\
\hline Daily menu evaluation & & $\mathrm{x}$ & $x$ & $\mathrm{X}$ & $\mathrm{X}$ \\
\hline Weighted Food Record & & $\mathrm{x}$ & $x$ & $x$ & $\mathrm{X}$ \\
\hline \multicolumn{6}{|c|}{ BIOCHEMICAL ASSESSMENTS } \\
\hline Intestinal Permeability test & & $\mathrm{x}$ & $x$ & $\mathrm{x}$ & $x$ \\
\hline Inflammatory markers & & $x$ & $x$ & $x$ & $x$ \\
\hline Vascular function markers & & $x$ & $x$ & $x$ & $x$ \\
\hline Oxidative stress markers & & $x$ & $x$ & $x$ & $x$ \\
\hline Metabolomics & & $x$ & $x$ & $x$ & $x$ \\
\hline Microbiota composition & & $x$ & $x$ & $\mathrm{X}$ & $x$ \\
\hline Blood microbiomics & & $x$ & $x$ & $x$ & $x$ \\
\hline
\end{tabular}

class III or IV NYHA - New York Heart Association)

- Antibiotic treatment in the last month

- Malignant tumor that required treatment in the previous 2 years

Each subject enrolled has been assigned to an ID number. The encoding of samples is hidden to both the investigators and the participants. All clinical and personal data, including the biological samples, of the subjects involved in the study are collected and stored anonymously.

\section{Polyphenol-rich dietary protocol}

In order to define the polyphenol-rich dietary protocol, an initial estimation of nutrient and total polyphenol intake was performed through the analysis of the daily menu provided at the OIC Foundation.

Subsequently, an identification of the specific polyphenol-rich food products to be included in the diet was carried out in order to consider not only the amount and contribution of the different polyphenols but also the food preparation in order to ensure their bioavailability. In addition, an evaluation of conditions to enable optimal texture (e.g. considering the use of purées instead of the whole product) and an assessment of the product acceptability by the target population was also undertaken.

The polyphenol-rich dietary protocol (PR-diet) was finally developed by including in the C-diet 3 portions per day of the following selected polyphenol-rich foods: berries and related products, blood orange, pomegranate, green tea, Renetta apple, and dark chocolate.

A schematic plan of the type and serving sizes of polyphenol-rich products consumed daily in the PR-diet is shown in Table 2. The MaPLE polyphenol-rich foods provided a mean of $724 \mathrm{mg} /$ day of total polyphenols as estimated by Folin-Ciocalteu analysis [26]. In addition, the PR-diet and C-diet were kept comparable in terms of energy intake and nutrient composition, and to achieve this, polyphenol-rich products were substitute for other comparable products (e.g. foods used for snack or breakfast) and this continued across the entire period of intervention. 
Table 2 Daily plan of MaPLE polyphenol-rich food products: 3 portions per day are scheduled. Legend: ${ }^{\circ}$ Chocolate powder was dissolved in hot milk or water; * Green tea was prepared by solubilization of $200 \mathrm{mg}$ of green tea extract in $200 \mathrm{ml}$ of hot water. ${ }^{+}$Renetta apple purée was prepared in controlled conditions and stored at $-18^{\circ} \mathrm{C}$.

\begin{tabular}{|c|c|c|c|}
\hline WEEK & \multicolumn{3}{|c|}{$\begin{array}{l}\text { POLYPHENOL-RICH FOOD PRODUCTS } \\
\text { (SERVINGS) }\end{array}$} \\
\hline MONDAY & $\begin{array}{l}\text { Chocolate callets } \\
\qquad(10 \mathrm{~g})\end{array}$ & $\begin{array}{l}{ }^{+} \text {Renetta apple purée } \\
\qquad(100 \mathrm{~g})\end{array}$ & $\begin{array}{l}\text { Pomegranate juice } \\
\qquad(125 \mathrm{ml})\end{array}$ \\
\hline TUESDAY & $\begin{array}{l}\text { Blood Orange } \\
\qquad(200 \mathrm{ml})\end{array}$ & $\begin{array}{l}\text { Renetta apple } \\
\qquad(150 \mathrm{~g})\end{array}$ & $\begin{array}{l}\text { Berry purée } \\
\text { (100 g) }\end{array}$ \\
\hline WEDNESDAY & $\begin{array}{l}\text { Cocoa powder } \\
(2 \mathrm{~g})\end{array}$ & $\begin{array}{l}\text { Renetta apple } \\
\quad(150 \mathrm{~g})\end{array}$ & $\begin{array}{l}\text { Blueberry } \\
(120 \mathrm{~g})\end{array}$ \\
\hline THURSDAY & $\begin{array}{l}\text { Green tea* } \\
\text { (200 ml) }\end{array}$ & $\begin{array}{l}\text { Chocolate callets } \\
\qquad(10 \mathrm{~g})\end{array}$ & $\begin{array}{l}\text { Renetta apple } \\
\qquad(150 \mathrm{~g})\end{array}$ \\
\hline FRIDAY & $\begin{array}{l}\text { Blood Orange } \\
\qquad(200 \mathrm{ml})\end{array}$ & $\begin{array}{l}\text { Pomegranate juice } \\
\qquad(125 \mathrm{ml})\end{array}$ & $\begin{array}{l}\text { Blueberry } \\
\text { (120 g) }\end{array}$ \\
\hline SATURDAY & $\begin{array}{l}\text { Chocolate callets } \\
\qquad(10 \mathrm{~g})\end{array}$ & $\begin{array}{l}\text { Berry purée } \\
\text { (100 g) }\end{array}$ & $\begin{array}{c}{ }^{+} \text {Renetta apple purée } \\
\text { (100 g) }\end{array}$ \\
\hline SUNDAY & $\begin{array}{l}\text { Green tea } \\
(200 \mathrm{ml})\end{array}$ & $\begin{array}{l}\text { Blood orange fruit } \\
\qquad(110 \mathrm{~g})\end{array}$ & $\begin{array}{l}\text { Renetta apple } \\
\quad(150 \mathrm{~g})\end{array}$ \\
\hline
\end{tabular}

\section{Information on potential adverse effects}

Even though no reports of adverse effects due to a polyphenol-rich diet had been registered or reported in the literature, subjects were advised to annotate and communicate any adverse symptom perceived during the intervention period. Since green tea was selected within the polyphenol-rich food sources to be used in the intervention study, there was a comprehensive discussion to define the dose to use. Green tea extract is a rich source of epigallocatechin-3-gallate (EGCG) known for many different protective effects; however, the intake of very high doses of EGCG/green tea extracts as supplements has been reported to cause liver toxicity. Recently, it has been proposed an EGCG upper level (UL) based on human intervention studies of $300 \mathrm{mg}$ EGCG/day in healthy adults [27]. The proposed UL based on an ADI derived from animal toxicity data was $322 \mathrm{mg}$ EGCG/day in a $70 \mathrm{~kg}$ adult. These values are applicable to the oral exposure under fed conditions, and consistent with those published by France [28] and Italy [29]. In MaPLE, the dietary intervention provided 200 $\mathrm{mg}$ of green tea powder (i.e. $120 \mathrm{mg}$ total polyphenol including about 100 mg EGCG) 2 times per week. This quantity was regarded as very likely to be safe taking into account the target population and the contribution of other food sources containing EGCC.

\section{Assessment of food intake}

Food intake before (enrollment phase) and during the intervention periods was recorded through the evaluation of OIC Foundation daily menus and the use of WFRs. The daily menus, covering different seasons, were analyzed to quantify nutrient and polyphenol content. Moreover, the day before each time-point, a WFR was completed and both nutrient and polyphenol intake were estimated. At least 3-WFRs were completed during each intervention period. Daily menus and WFRs were 
assessed using MetaDieta ${ }^{\circ}$ (Me.Te.Da S.r.l., San Benedetto del Tronto, Italy) to estimate energy and nutrient intake. Total polyphenol estimation was performed by using the Phenol Explorer database (phenol-explorer.eu) to provide estimates of polyphenol concentrations in each food, and where there were no useful values, using our proprietary data or values obtained from the literature. Total polyphenol content of the foods was estimated directly using the Folin-Ciocalteau method [30].

\section{Biological sampling}

Blood, urine and fecal samples were collected at each timepoint as defined in Fig. 1. For blood drawing, a specific vacutainer was used. Urine and fecal samples were collected using specific containers designed for this purpose. An aliquot of each collected blood sample was immediately stored at $-80^{\circ} \mathrm{C}$ for microbiomic analyses. The remaining blood was processed by centrifugation and then serum and peripheral blood mononuclear cell (PBMC) fractions were obtained, divided into aliquots and stored at $80^{\circ} \mathrm{C}$. Urine and fecal sample were divided into aliquots, and all human tissue samples were stored at $-80^{\circ} \mathrm{C}$ until analysis.

In addition, a brush was used to collect an oral mucosal sample from each participant for further evaluation. The brush with the collected tissue was stored in a cryovial containing a buffered saline solution, which was immediately frozen.

\section{Outcome measurements}

The primary selected outcome of the study was zonulin as an IP marker, whereas other IP related markers (e.g. CD14, calprotectin), inflammatory markers (CRP, TNF$\alpha$, IL-6), oxidative stress and vascular function markers (DNA damage, VCAM-1, ICAM-1), metabolomics and microbiomics (16S rRNA gene quantification and taxonomic profiling) were included as secondary outcomes to support and validate our study hypothesis.

\section{Anthropometric measurements}

Body weight, height and BMI calculation were assessed at the beginning and the end of each intervention period following the international guidelines of Lohman et al. [31].

\section{Blood pressure}

Each participant was monitored at the beginning and the end of each intervention period measuring both systolic and diastolic pressure obtained in a resting, seated position following the validated JNC 7 guidelines [32].

\section{Metabolic and functional markers}

At enrollment and at each time-point, metabolic and functional parameters (i.e. glucose, insulin, lipid profile, liver and renal function) were assessed by a standardized validated protocol, using an automatic biochemical analyzer (ILAB 650, Instrumentation Laboratory, Lexington, MA). Low density lipoprotein cholesterol (LDL-C) concentration was estimated using the Friedewald formula [33], while non-high density lipoprotein cholesterol (non-high density lipoprotein-cholesterol, HDL-C) was calculated by subtracting HDL-C from total cholesterol (TC). The HOMA-Index and Cockroft-Gault index were calculated according to the relevant formula [34, 35].

\section{Intestinal permeability evaluation}

Intestinal permeability was evaluated by quantifying serum zonulin concentrations. Human zonulin is a protein (i.e. prehaptoglobin-2) released by enterocytes able to promote the activation of the signaling transduction pathway that cause tight junction protein disassembly enabling potential bacterial factor translocation [36]. In this study, zonulin serum levels were quantified using the Immunodiagnostik ${ }^{\bullet}$ ELISA kit (Bensheim, Germany) with samples collected in the selection phase and at the beginning and the end of each intervention period. Subjects selection based on IP was performed by considering reference values reported in the manufacturer's instructions and data published on different target groups [37-39]. Other IP related markers, such as serum CD14 and fecal calprotectin, were also quantified to support the primary outcome.

\section{Inflammatory markers}

The concentrations of several markers related to inflammatory processes were quantified using specific ELISA kits (R\&D Systems, Biotechne, Abingdon, UK). CRP (DCRP00), IL-6 (HS600B), TNF- $\alpha$ (HSTA00E) were quantified in serum at the beginning and the end of each intervention periods.

\section{Vascular function markers}

In order to assess vascular function, vascular cell adhesion molecule-1 (VCAM-1) and intercellular adhesion molecule-1 (ICAM-1) were quantified in serum samples at each intervention time point using an ELISA kit (Booster ${ }^{\bullet}$ from Vinci Biochem S.r.l., Vinci, Italy).

\section{Oxidative stress marker (comet assay)}

The levels of endogenous and oxidatively-induced DNA damage, as markers of oxidative stress, were assessed in PBMCs by the comet assay. The samples are collected before and after each intervention period. Levels of endogenous DNA damage were assessed using a specific enzyme (formamidopyrimidine DNA glycosylase, FPG sensitive sites) that can be used to detect 8-oxo-7,8-dihydro- 2 '-deoxyguanosine (8-oxodG) and ring-opened formamidopyrimidine nucleobases. Oxidatively-induced DNA damage was measured by treating the cells with 
hydrogen peroxide and by evaluating the capacity of cells to counteract an oxidative insult. Both Comet assay protocols have been previously described by Del Bo' et al. [32].

\section{Blood bacterial load and taxonomic profiling}

Bacterial DNA quantification and sequencing reactions were performed by Vaiomer SAS (Labège, France) using optimized blood-specific techniques as described earlier [40-43]. Specifically, DNA was extracted from $100 \mu \mathrm{l}$ of whole blood and quantified by quantitative PCR targeting the V3-V4 hypervariable regions of the bacterial $16 \mathrm{~S}$ rRNA gene with primers EUBF 5'-TCCTACGGGA GGCAGCAGT-3' and EUBR 5' -GGACTACCAGGGT ATCTAATCCTGTT-3' [44]. The results are reported as 16S rRNA gene copies per ng of total DNA and per $\mu \mathrm{l}$ of blood. DNA from whole blood was also used for $16 \mathrm{~S}$ rRNA gene taxonomic profiling using MiSeq Illumina ${ }^{\circ}$ technology $(2 \times 300$ paired-end MiSeq kit V3, set to encompass 467-bp amplicon) as previously described $[42,43]$. To determine bacterial community profiles, the bar-coded Illumina paired reads were demultiplexed, then single read sequences were trimmed and paired for each sample independently into longer fragments; nonspecific amplicons ( $<350$ bases or $>500$ bases) were removed and remaining sequences clustered into operative taxonomic units (OTUs) using FROGS v1.4.0 [45] with default parameters; a taxonomic assignment was finally performed against the Silva 128 Parc database. Bioinformatics analysis of the sequencing data was also performed using the Quantitative Insights Into Microbial Ecology (QIIME) pipeline [46].

\section{Fecal microbiota composition}

All of the following steps were performed in-house at QIB. Fecal samples were weighed into Lysing Matrix E bead beating tubes (MPBio, Santa Ana, CA, USA) and extraction was completed according to the manufacturer's protocol for the FastDNA ${ }^{\text {max }}$ SPIN Kit for Soil (MPBio) but extending the bead beating time to 3x60s. DNA was quantified using a Qubit 2.0 fluorometer (Invitrogen, Carlsbad CA, USA), normalized to $5 \mathrm{ng} / \mu \mathrm{l}$ and the V3/V4 region of the $16 \mathrm{~S}$ rRNA was amplified using the primers detailed below. Sequencing was performed using a 600 cycle MiSeq v3 reagent kit (Illumina, San Diego, CA, USA) giving approximately 100,000 reads per sample.

Bioinformatic analysis was conducted using VSEARCH [47]; reads were merged, and primer sequences trimmed. Reads were dereplicated and singletons removed. Prior to Chimera removal, reads were clustered at $97 \%$ similarity, de novo Chimera removal was performed using the UCHIME algorithm [48] and the OTU table and sequences were prepared. Data was subsequently analyzed using the phyloseq package in $\mathrm{R}$ [49].

Primers:

16S 341F - TCGTCGGCAGCGTCAGATGTGTATA AGAGACAGCCTACGGGNGGCWGCAG.

16S 806R - GTCTCGTGGGCTCGGAGATGTGTA TAAGAGACAGGACTACHVGGGTATCTAATCC.

In addition, taxonomic profiling was carried out through shotgun sequencing. In brief, metagenomic DNA isolated from fecal samples was sequenced using an HiSeq instrument (Illumina, San Diego, CA) by CosmosID (Rockville, MD, USA). Microbial community composition was determined through the analysis of shotgun metagenomic datasets with the CosmosID metagenomic software as previously described [50].

\section{Metabolomics}

Urine samples collected prior and after each intervention period were subjected to targeted metabolomics analysis by applying the Quantitative Dietary Fingerprinting approach recently developed by González-Domínguez et al. [51] with the aim of monitoring metabolite alterations derived from the polyphenol-rich diet and to associate these changes with improvements in clinical and biochemical outcome measurements (e.g. IP evaluated through zonulin levels, inflammatory and oxidative stress markers, blood bacterial load). To this end, urine samples were treated by solid phase extraction (SPE) and subsequently analyzed by reversed-phase ultra-highperformance liquid chromatography coupled to tandem mass spectrometry (RP-UHPLC-MS/MS) to obtain a comprehensive assessment of the urinary food metabolome, with the simultaneous quantitative determination of about 350 dietary derived metabolites. Complementarily, plasma samples are also analyzed using a modification of the previously described targeted metabolomics approach, adapted to deal with the chemical complexity of blood samples (high content of proteins and lipids) and to enlarge the metabolomic coverage. This novel method is based on a similar RP-UHPLC-MS/MS instrumental configuration that enables the simultaneous measurement of both food intake biomarkers and endogenous metabolites from multiple chemical classes (ca. 1000 metabolites), including amino acids and derivatives, biogenic amines, carbohydrates, organic and fatty acids, vitamins and various lipid classes (e.g. acylcarnitines, steroid hormones, bile acids), among others. To expand the method coverage towards the high-polarity low molecular weight metabolome, an orthogonal hydrophilic interaction liquid chromatography (HILIC) procedure was also applied, covering a broad range of polar metabolites (ca. 300 metabolites), comprising common and acetylated amino acids and microbiota derivatives, low molecular weight organic acids (including 
short chain fatty acids and related compounds) and carbohydrates (e.g. sugars, conjugates and advanced glycation end products).

\section{Sample size, randomisation, and statistics}

According to data literature $[38,52]$ it was estimated that 50 subjects were required to demonstrate an IP reduction of $30 \%$ with $80 \%$ power and 0.05 significance and considering a $15 \%$ drop-out rate. Subjects were randomly divided by using a computer random number generator. The randomisation and allocation were performed by a person not involved in the trial and blinded to the participants, investigators/health care providers and researchers involved in samples analysis. Statistical analyses were performed by means of $\mathrm{R}$ statistic software version 3.4.2. Particularly, the following statistical elaborations will be performed to identify significant differences between treatments: (i) the analysis of variance (ANOVA) with repeated measures, (ii) Wilcoxon paired data test, (iii) Linear Mixed Model (LMM) analysis. In addition, regression and correlation analyses (Spearman and Kendal test) are carried out to highlight associations between blood microbiomic data, fecal bacterial profiling data, and physiological and biochemical data. When appropriate, a post-hoc $p$-value adjustment is performed using the Hochberg-Benjamin correction. Significance is set at $P \leq 0.05$; significance in the range $0.05<P<0.10$ is accepted as trend. Potential gender differences will be also considered in all the analyses.

\section{Discussion}

There is growing evidence of a link between IP impairment and increased inflammation [2]. Since aging is characterized by low grade systemic inflammation it is possible that an increase in IP may induce the activation of inflammatory pathways and the immune system caused by the translocation of intestinal microbes, toxins, and/or nutritional components from the gut lumen through the epithelium and into the bloodstream [52]. While there is preliminary mechanistic evidence obtained in animal models on the complex interaction between age-associate microbial dysbiosis, IP and inflammation [5], the properties of the human intestinal barrier, in the context of the ageing process, has not been fully investigated [4]. The dietary pattern and the intestinal microbial ecosystem homeostasis have been addressed as potential key points for the development of strategies to enable healthy aging. The manipulation and/or improvement of the diet by increasing the consumption of food bioactives (e.g. polyphenols) or specific nutrients is recognized as a potential powerful tool to be explored also in the context of IP. However, human intervention studies are still very scarce, and most of these performed using probiotics, prebiotic fibers and dietary supplements [21].

By considering this premise, the MaPLE RCT here described aimed to investigate whether a PR rich-diet can improve the intestinal microbial ecosystem of older subjects characterised by an increased IP. In addition, it is hypothesized that such modulation could promote an overall beneficial impact on IB function, a decreased IP and translocation of inflammogenic bacterial factors in the blood.

The development and management of well-controlled and adequately balanced dietary intervention studies is not an easy task and it becomes even more difficult when the target population is older subjects. Consequently, the first task of the project was dedicated to the optimization of the trial in order to overcome the possible problems related to compliance with the dietary instructions and to other relevant potential confounding factors (e.g. periods of illness or the use of drugs that may be relevant in this target group). For this reason, the MaPLE RCT was planned in a residential area for older people, since it provided a favourable and controlled environment in which it was possible to optimize and standardize most of the important experimental conditions. For example, since outcome data from dietary intervention studies are prone to being affected by individual differences in diets and lifestyle behaviour over time (e.g. during the two eight week periods of dietary intervention), we were able to ensure both strict compliance with the dietary intervention and a consistent dietary pattern among participants by including the polyphenol-rich products in their usual meals provided by the residential home. In addition, the selection of polyphenol-rich foods was based on three important considerations: (i) That the types of foods selected were largely universally liked, (ii) that the texture of the selected products was suitable for older subjects (e.g. with dentition challenges), and (iii) that the portion of food would reliably provide a high dose of polyphenols. In addition, weighed food intake was also assessed to provide us with data to allow accurate estimates of actual nutrient and polyphenol intake in the two periods of treatment (PR- and C- diet). This allowed a high degree of control and substantially reduced between treatments differences.

As regard the primary outcome, serum zonulin concentrations were used as the marker of IP because of the low reliability and applicability of the multi-sugar test in the older population (i.e. due to a high rate of incontinence amongst the elderly participants and the need for adherence to a strict dietary protocol before the test) [52].

It is also noteworthy that the MaPLE RCT is testing, for the first time, the hypothesis that a dietary intervention may modulate quantitatively the bacterial DNA in bloodstream and qualitatively the blood microbiota 
composition. This should provide further evidence of the impact of the dietary intervention on IP being potentially associated with a reduction in translocation of bacterial factors. Other objectives of the MaPLE RCT are to integrate microbiota profiling data with inflammation and metabolomics data to improve understanding on the impact of the dietary intervention. In addition, the interindividual response to the treatment will be investigated and food metabolite profiling data will be exploited for the identification of a set of potential biomarkers with relevance in the context of preventing or treating impaired IP.

Finally, results will be pivotal for the development of new dietary approaches and guidelines for managing IP related conditions in the complex context of healthy aging.

\section{Abbreviations}

IP: Intestinal permeability; MaPLE: Microbiome mAnipulation through Polyphenols for managing Leakiness in the Elderly; PR-diet: Polyphenol-rich diet; C-diet: Control diet; WFRs: Weighted food records; MNA: Mini nutritional assessment; MMSE: Mini mental state examination; COPD: Chronic obstructive pulmonary disease; NYHA: New York heart association; EGCG: Epigallocatechin-3-gallate; UL: Upper level; PBMCs: Peripheral blood mononuclear cells; LDL-C: lipoprotein cholesterol; HDL-C: Non-high density lipoprotein-cholesterol; TC: Total cholesterol; VCAM-1: Vascular cell adhesion molecule-1; ICAM-1: Intercellular adhesion molecule-1; 8-oxodG: 8-oxo-7,8dihydro-2'-deoxiguanosine; QIIME: Quantitative insights into microbial ecology; SPE: Solid phase extraction; HILIC: Orthogonal hydrophilic interaction liquid chromatography; ANOVA: Analysis of variance; LMM: Linear mixed model

\section{Acknowledgments}

The authors are grateful to the in-kind contribution of products used in the dietary intervention study: in particular Barry Callebaut, Indena, Melinda, Oranfrizer, Roberts Berrie, Zuegg. We thank Giulia Minto and Nicola Fassetta for their help and contribution during the intervention period. We warmly thank all the volunteers participating to the study for their valuable contribution.

We are grateful to Alberto Fantuzzo, Chiara Cavazzini, Lorella Pinton, Paolo Bergantin, Rosanna Ceccato, Pamela Soranzo, and Silvana Giraldini at Opera Immacolata Concezione (OIC Foundation, Padua, Italy) for their coordinating activities in the nursing home. We are also grateful to all physicians (Michela Rigon, Lorena D’Aloise, Antonio Merlo, Elisabetta Bernardinello, Nadia Malacarne, Silvana Bortoli, Fabiola Talato, Agostino Corsini, Maria Licursi, Nicoletta Marcon, Angela Sansone), nurses and other personnel at OIC who were essential to complete the study successfully. The Department of Food, Environmental and Nutritional Sciences, Università degli Studi di Milano, partially covered the open access APC.

\section{Authors' contributions}

MaPLE project includes three research units: (i) University of Milan, Italy, coordinated by Patrizia Riso (PR) and Simone Guglielmetti (SG), who are the principal investigators of the project, (ii) University of Barcelona, Spain, coordinated by Cristina Andres-Lacueva (CAL), and (iii) Quadram Institute, UK, coordinated by Paul A Kroon (PAK).

All listed authors meet the ICMJE criteria for authorship. In particular, PR and SG are responsible for the trial conception and design. AC contributed to the development of study protocol for clinical and ethical aspects and to the selection of biochemical markers under study from a clinical perspective. CAL and PAK contributed to the definition of polyphenol-rich dietary intervention and markers selection. CDB contributed to the development of the study protocol and with PR, SG and SB drafted the first version of the manuscript. SG and GG contributed to the definition of the trial protocol enabling the study of blood microbiomic and metagenomic. RGD and GP coordinated by CAL implemented the study protocol to enable metabolomics approach. MP, NHL and RZR contributed to the definition of the design needed to enable accurate dietary intake assessment. BK and
MSW contributed to the development of protocol aspects defined for microbiota composition evaluation and inflammation coordinated by PAK who also set the markers for polyphenol metabolism evaluation. All the authors critically revised the draft and approved the final version.

\section{Funding}

This work was completed as part of the MAPLE project (Gut and Blood Microbiomics for Studying the Effect of a Polyphenol-Rich Dietary Pattern on Intestinal Permeability in the Elderly) supported within the European Joint Programming Initiative "A Healthy Diet for a Healthy Life" (JPI HDHL, http:// www.healthydietforhealthylife.eu/) granted by Mipaaft (Italy, D.M. 8245/7303/ 2016), MINECO (Spain, PCIN-2015-238), and BBSRC (U.K., BB/R012512/1). Cristina Andres-Lacueva was also supported by grant 2017SGR1546 from the Generalitat de Catalunya's Agency AGAUR, funds from CIBERFES (co-funded by the FEDER Program from the European Union), and ICREA Academia Award 201. Additional funding was provided by the Biotechnology and Biological Sciences Research Council (UK) through an Institute Strategic Programme Grant ('Food Innovation and Health'; Grant No. BB/R012512/1) to the Quadram Institute Bioscience. Raúl González-Domínquez thanks the "Juan de la Cierva" program from MINECO (FJCl-2015-26590).

The sponsors of this work have no role in the development of study protocol, methods, data collections and analyses, and the preparation of the manuscript.

\section{Availability of data and materials}

At the end of the project, after the final elaborations, the datasets generated during the study will be made freely available in the Dataverse repository, https://dataverse.unimi.it/dataverse/JPI-MaPLE.

\section{Ethics approval and consent to participate}

The Ethics Committee of the Università degli Studi di Milano approved the study protocol (15/02/2016; ref.: 6/16/CE_15.02.16_Verbale_All-7). All participants gave their written informed consent to participate in the MaPLE RTC allowing also the use of samples and data collected within the trial.

\section{Consent for publication}

Not applicable.

\section{Competing interests}

The authors declare that they have no competing interests.

\section{Author details}

'Department of Food, Environmental and Nutritional Sciences (DeFENS), Università degli Studi di Milano, 20133 Milan, Italy. ${ }^{2}$ Geriatria, Accettazione Geriatrica e Centro di Ricerca per I'Invecchiamento, IRCCS INRCA, 60127 Ancona, Italy. ${ }^{3}$ Biomarkers and Nutrimetabolomics Laboratory, Department of Nutrition, Food Sciences and Gastronomy, Food Technology Reference Net (XaRTA), Nutrition and Food Safety Research Institute (INSA), Faculty of Pharmacy and Food Sciences, University of Barcelona, 08028 Barcelona, Spain. ${ }^{4}$ CIBER de Fragilidad y Envejecimiento Saludable (CIBERfes), Instituto de Salud Carlos III, 08028 Barcelona, Spain. ${ }^{5}$ Unit of Nutrition and Cancer, Cancer Epidemiology Research Programme, Catalan Institute of Oncology (ICO), Bellvitge Biomedical Research Institute (IDIBELL), Barcelona, Spain. ${ }^{6}$ Quadram Institute Bioscience, Norwich Research Park, Norwich NR4 7UQ, UK.

Received: 13 September 2019 Accepted: 12 February 2020

Published online: 26 February 2020

\section{References}

1. Vancamelbeke $M$, Vermeire $S$. The intestinal barrier : a fundamental role in health and disease. Expert Rev Gastroenterol Hepatol. 2017;11(9):821-34.

2. Bischoff SC, Barbara G, Buurman W, Ockhuizen T, Schulzke JD, Serino M, et al. Intestinal permeability - a new target for disease prevention and therapy. BMC Gastroenterol. 2014;14(1):1-25.

3. Meier J, Sturm A. The intestinal epithelial barrier: does it become impaired with age? Dig Dis. 2009;27(3):240-5.

4. Britton E, McLaughlin JT. Ageing and the gut. Proc Nutr Soc. 2013;72(1):173-7.

5. Camilleri M, Madsen K, Spiller R, Van Meerveld BG, Verne GN. Intestinal barrier function in health and gastrointestinal disease. Neurogastroenterol Motil. 2012;24(10):976. 
6. Heyman M, Abed J, Lebreton C, Cerf-Bensussan N. Intestinal permeability in coeliac disease: insight into mechanisms and relevance to pathogenesis. Gut. 2012;61(9):1355-64.

7. Arthur JC, Perez-Chanona E, Mühlbauer M, Tomkovich S, Uronis JM, Fan TJ, et al. Intestinal inflammation targets cancer-inducing activity of the microbiota. Science (80- ). 2012;338(6103):120-3.

8. Martin CR, Osadchiy V, Kalani A, Mayer EA. The brain-gut-microbiome Axis. CMGH. 2018;6(2):133-48.

9. Malago J. Contribution of microbiota to the intestinal physicochemical barrier. Benef Microbes. 2015;6(3):295-3.

10. Kiesslich R, Goetz M, Angus EM, Hu Q, Guan Y, Potten C, et al. Identification of epithelial gaps in human small and large intestine by confocal Endomicroscopy. Gastroenterology. 2007;133(6):1769-78.

11. Queipo-Ortuno Ml, Boto-Ordonez M, Murri M, Gomez-Zumaquero JM, Clemente-Postigo M, Estruch R, et al. Influence of red wine polyphenols and ethanol on the gut microbiota ecology and biochemical biomarkers. Am J Clin Nutr. 2012 Jun;95(6):1323-34.

12. Vendrame S, Guglielmetti S, Riso P, Arioli S, Klimis-Zacas D, Porrini M. Six-week consumption of a wild blueberry powder drink increases Bifidobacteria in the human gut. J Agric Food Chem. 2011;59(24):12815-20.

13. Tzounis X, Rodriguez-Mateos A, Vulevic J, Gibson GR, Kwik-Uribe C, Spencer JPE. Prebiotic evaluation of cocoa-derived flavanols in healthy humans by using a randomized, controlled, double-blind, crossover intervention study. Am J Clin Nutr. 2011;93(1):62-72.

14. Koeth RA, Wang Z, Levison BS, Buffa JA, Org E, Sheehy BT, et al. Intestinal microbiota metabolism of I-carnitine, a nutrient in red meat, promotes atherosclerosis. Nat Med. 2013;19(5):576-85.

15. Ferrario C, Taverniti V, Milani C, Fiore W, Laureati M, De Noni I, et al. Modulation of fecal Clostridiales Bacteria and butyrate by probiotic intervention with lactobacillus paracasei DG varies among healthy adults. J Nutr. 2014;144(11):1787-96.

16. Plöger S, Stumpff F, Penner G, Schulzke J, Gäbel G, Martens H, et al. Microbial butyrate and its role for barrier function in the gastrointestinal tract. Ann N Y Acad Sci. 2012;1258:52-9.

17. Wada M, Tamura A, Takahashi N, Tsukita S. Loss of claudins 2 and 15 from mice causes defects in paracellular $\mathrm{Na}+$ flow and nutrient transport in gut and leads to death from malnutrition. Gastroenterology. 2013;144(2):369-80.

18. Claesson MJ, Jeffery IB, Conde S, Power SE, O'connor EM, Cusack S, et al. Gut microbiota composition correlates with diet and health in the elderly. Nature. 2012;488(7410):178-84.

19. Tiihonen K, Ouwehand AC, Rautonen N. Human intestinal microbiota and healthy ageing. Ageing Res Rev. 2010;9(2):107-16.

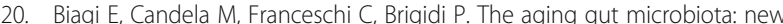
perspectives. Ageing Res Rev. 2011;10(4):428-9.

21. Bernardi S, Del Bo' C, Marino M, Gargari G, Cherubini A, Andres-Lacueva C, et al. Polyphenols and intestinal permeability: rationale and future perspectives. J Agric Food Chem. 2019. https://doi.org/10.1021/acs.jafc.9b02283.

22. Peron G, Hidalgo-Liberona N, González-Domínguez R, Garcia-Aloy M, Guglielmetti S, Bernardi S, et al. Exploring the Molecular Pathways behind the Effects of Nutrients and Dietary Polyphenols on Gut Microbiota and Intestinal Permeability: A Perspective on the Potential of Metabolomics and Future Clinical Applications. J Agric Food Chem. 2019. https://doi.org/10.1021/acs.jafc.9b01687.

23. Boto-Ordonez M, Urpi-Sarda M, Queipo-Ortuno MI, Tulipani S, Tinahones FJ, Andres-Lacueva C. High levels of Bifidobacteria are associated with increased levels of anthocyanin microbial metabolites: a randomized clinical trial. Food Funct. 2014 Aug;5(8):1932-8.

24. Cardonaa F, Andrés-Lacuevac C, Tulipania S, Tinahonesb FJ. María Isabe Queipo-Ortuño. Benefits of polyphenols on gut microbiota and implications in human health. J Nutr Biochem. 2013;24:1415-22.

25. Zamora-ros R, Cherubini A, Urp M, Bandinelli S, Ferrucci L, Andres-lacueva C. High concentrations of a urinary biomarker of polyphenol intake are associated with decreased mortality in older adults. J Nutr. 2013;143:1445-50.

26. Agbor AG, Vinson JA, Donnelly PE. Folin-Ciocalteau reagent for Polyphenolic assay. Int J Food Sci Nutr Diet. 2014;3(8):147-56.

27. Yates AA, Erdman JW, Shao A, Dolan LC, Griffiths JC. Bioactive nutrients time for tolerable upper intake levels to address safety. Regul Toxicol Pharmacol. 2017:84:94-101.

28. French Ministry of Health. Decree Establishing the List of Plants, Other than Fungi, Authorized in Food Supplements and the Conditions of Their Employment, F.R. Off J French Repub. 2014;
29. Italian Ministry of Health 2016. Other Nutrients and Other Substances with Nutritional or Physiological Effects. Dir Heal Saf Food Nutr Available athttp// www.salute.gov.it/imgs/C_17_EventiStampa_355_intervisteRelatori_ itemInterviste 1 fileAllegatolntervista.pdf. 2016:

30. Neveu V, Perez-Jiménez J, Vos F, Crespy V, du Chaffaut L, Mennen L, et al. Phenol-Explorer: an online comprehensive database on polyphenol contents in foods. Database (Oxford). 2010;2010 Article ID bap024.

31. Seefeldt VD, Harrison GG. In: Anthropometric Standardization Reference Manual, Lohman TG, Roche AF, Martorell R, Eds. Champaign: Human Kinetics Books; 1988: pg 111.

32. Chobanian AV, Bakris GL, Black HR, Cushman WC, Green LA, Izzo JL, et al. Seventh report of the joint National Committee on prevention, detection, evaluation, and treatment of high blood pressure Hypertension. 2003;42(6):1206-52.

33. Knopfholz J, Disserol CCD, Pierin AJ, Schirr FL, Streisky L, Takito LL, et al. Validation of the friedewald formula in patients with metabolic syndrome. Cholesterol. 2014;2014:261878.

34. Drinka PJ, Langer E. The Cockroft-Gault formula. J Am Geriatr Soc. 1989;37(8):820.

35. Antuna-Puente B, Disse E, Rabasa-Lhoret R, Laville M, Capeau J, Bastard JP. How can we measure insulin sensitivity/resistance? Diabetes Metab. 2011;37(3):179-88

36. Sturgeon C, Fasano A. Zonulin, a regulator of epithelial and endothelial barrier functions, and its involvement in chronic inflammatory diseases. Tissue Barriers. 2016;4(4):e1251384.

37. Ciccia F, Guggino G, Rizzo A, Alessandro R, Luchetti MM, Milling S, et al. Dysbiosis and zonulin upregulation alter gut epithelial and vascular barriers in patients with ankylosing spondylitis. Ann Rheum Dis. 2017;76(6):1123-32.

38. Moreno-Navarrete JM, Sabater M, Ortega F, Ricart W, Fernández-Real JM. Circulating zonulin, a marker of intestinal permeability, is increased in association with obesity-associated insulin resistance. PLoS One. 2012;7(5):e37160.

39. Li C, Gao M, Zhang W, Chen C, Zhou F, Hu Z, et al. Zonulin regulates intestinal permeability and facilitates enteric Bacteria permeation in coronary artery disease. Sci Rep. 2016;6:29142.

40. Amar J, Lange C, Payros G, Garret C, Chabo C, Lantieri O, et al. Blood Microbiota Dysbiosis Is Associated with the Onset of Cardiovascular Events in a Large General Population: The D.E.S.I.R. Study. PLoS One. 2013;8(1):e54461.

41. Lelouvier B, Servant F, Païssé S, Brunet AC, Benyahya S, Serino M, et al. Changes in blood microbiota profiles associated with liver fibrosis in obese patients: a pilot analysis. Hepatology. 2016;64(6):2015-27.

42. Lluch J, Servant F, Païssé S, Valle C, Valière S, Kuchly C, et al. The characterization of novel tissue microbiota using an optimized $16 \mathrm{~S}$ metagenomic sequencing pipeline. PLoS One. 2015;10(11):e0142334.

43. Païssé S, Valle C, Servant F, Courtney M, Burcelin R, Amar J, et al. Comprehensive description of blood microbiome from healthy donors assessed by 165 targeted metagenomic sequencing. Transfusion. 2016;56(5):1138-47.

44. Nadkarni MA, Martin FE, Jacques NA, Hunter N. Determination of bacterial load by real-time PCR using a broad-range (universal) probe and primers set. Microbiology. 2002;148(Pt 1):257-66.

45. Escudié F, Auer L, Bernard M, Mariadassou M, Cauquil L, Vidal K, et al. FROGS: find, rapidly, OTUs with galaxy solution. Bioinformatics. 2018;34(8):1287-94.

46. Bolyen E, Rideout JR, Dillon MR, et al. Reproducible, interactive, scalable and extensible microbiome data science using QIIME 2. Nat Biotechnol. 2019; 37(8):852-7.

47. Rognes T, Flouri T, Nichols B, Quince C, Mahé F. VSEARCH: a versatile open source tool for metagenomics. PeerJ. 2016;18(4):e2584.

48. Edgar RC, Haas BJ, Clemente JC, Quince C, Knight R. UCHIME improves sensitivity and speed of chimera detection. Bioinformatics. 2011;27(16):2194-200.

49. McMurdie PJ, Holmes S. Phyloseq: an R package for reproducible interactive analysis and graphics of microbiome census data. PLoS One. 2013;8(4):e61217.

50. Hasan NA, Young BA, Minard-Smith AT, et al. Microbial community profiling of human saliva using shotgun metagenomic sequencing. PLoS One. 2014; 9(5):e97699.

51. González-Domínguez R, Urpi-Sarda M, Jáuregui O, Needs PW, Kroon PA, Andrés-Lacueva C. Quantitative Dietary Fingerprinting (QDF)—A Novel Tool for Comprehensive Dietary Assessment Based on Urinary Nutrimetabolomics. J Agric Food Chem. 2019. https://doi.org/10.1021/acs.jafc.8b07023.

52. Valentini $L$, Ramminger $S$, Haas V, Postrach E, Werich M, Fischer A, et al. Small intestinal permeability in older adults. Physiol Rep. 2014;2(4):e00281.

\section{Publisher's Note}

Springer Nature remains neutral with regard to jurisdictional claims in published maps and institutional affiliations. 